\title{
Successful treatment of pediatric intervertebral disc calcification using ultrasound-guided cervical root block
}

\author{
Hyungtae Kim ${ }^{1}$, Jae Wook Song ${ }^{1}$, and Yeon-Dong Kim ${ }^{2}$ \\ Department of Anesthesiology and Pain Medicine, ${ }^{1}$ Presbyterian Medical Center, University of Seonam College of Medicine, Jeonju, \\ ${ }^{2}$ Wonkwang University School of Medicine, Iksan, Korea
}

A 12-years-old boy (165 cm/72 kg) complained of severe posterior neck pain radiating to the right arm. The resting pain score was VAS 80 of 100 and on moving the neck the VAS was 90/100. X-ray and MRI showed C7-T1 intervertebral disc calcification (IDC) with right C8 nerve compression (Fig. 1). Conservative treatment such as, pain medication, physical therapy, and trigger point injection administered at another hospital, failed to relive his pain. Operation was recommended but parental consent was denied. The patient consulted our clinic for pain management. Laboratory finding were normal, and physical examination showed no neurological signs, with non specific electromyography. The severe pain was unresponsive to conservative treatment. We decided to perform cervical root block (CRB) instead of epidural block that was not suitable to the location, and caused fear in the pediatric patient.

Dual-guided CRB was performed with the patient in the left lateral position, after pre-scanning with ultrasound. We surgically disinfected both cervical areas and protected the ultrasound probe and cable with a sterile ultrasound probe cover (SonoLab ${ }^{\mathrm{TM}}$ $18 \times 120 \mathrm{~cm}$, Lucky Medical, Seoul, Korea). Right sided 8th CRB was performed with a portable ultrasound instrument, and a $50 \mathrm{~mm}$ linear 15-6 MHz probe (SonoSite M-Turbo ${ }^{\mathrm{TM}}$, SonoSite Inc., Bothell, WA, USA). The 8th CRB was performed as follows: The 5th, 6th, 7 th and 8 th cervical roots were identified by scanning from the supraclavicular area to the 6th transverse process. The skin was infiltrated with $1 \mathrm{ml}$ of lidocaine $1 \%$. A nerve stimulating needle with $30^{\circ}$ bevel (22-guage, $50 \mathrm{~mm}$, Stimuplex ${ }^{\circledR}$ D, B.Braun, Melsungen, Germany) was inserted un- der dual-guidance. We used $2 \mathrm{~Hz}$ stimulation frequency and an impulse duration of $0.1 \mathrm{~ms}$. The intensity of the stimulating current initially set to deliver $1.5 \mathrm{~mA}$, was decreased to obtain the minimal stimulating current. The needle was repositioned until the minimal stimulating current was $\leq 0.3 \mathrm{~mA}$ (Fig. 1). Five $\mathrm{ml}$ ropivacaine $0.2 \%$ with $10 \mathrm{mg}$ of triamcinolone acetate was injected surrounding the 8th nerve root after careful air bubble removal and an aspiration test.

The block was performed 4 times during 2 weeks. VAS was improved by $50 \%$ after the first block and the VAS was 20/100 after the 4 th procedure. The patient was discharged after 2 weeks since there was no pain. Subsequently, pain relief was with aceclofenac $100 \mathrm{mg}$ for 2 weeks. We did not administer further medication or nerve block. The patient had no pain and neurologic symptoms at the 2 month follow-up visit.

Pediatric IDC is a rare disorder without any clear etiologies and mechanisms. Calcification is mostly reported at the level of C6-7, but it may occur at any location [1]. IDC has a higher incidence in males, aged 7 to 8 years [1]. Various clinical symptoms have been reported. Neck pain and stiffness are most common. Low fever, limitation of motion, and muscle spasm are also commonly reported [1]. Neurologic symptoms appear due to compression of the nerve root and spinal cord from herniation, as a result of calcification. Signs of neurologic deficiency, including weakness, sensory loss, and myelopathy are rare [1]. However, $20 \%$ of patients exhibit neurologic symptoms due to epidural calcifications. In this case, the patient complained of severe pain, resulting from irritation of the cervical root by the irritants from

Corresponding author: Hyungtae Kim, M.D., Department of Anesthesiology and Pain Medicine, Presbyterian Medical Center, University of Seonam College of Medicine, 365, Seowon-ro, Wansan-gu, Jeonju 560-750, Korea. Tel: 82-63-230-1593, Fax: 82-63-230-8128, E-mail: ingwei2475@naver.com

(c) This is an open-access article distributed under the terms of the Creative Commons Attribution Non-Commercial License (http:// creativecommons.org/licenses/by-nc/3.0/), which permits unrestricted non-commercial use, distribution, and reproduction in any medium, provided the original work is properly cited. 

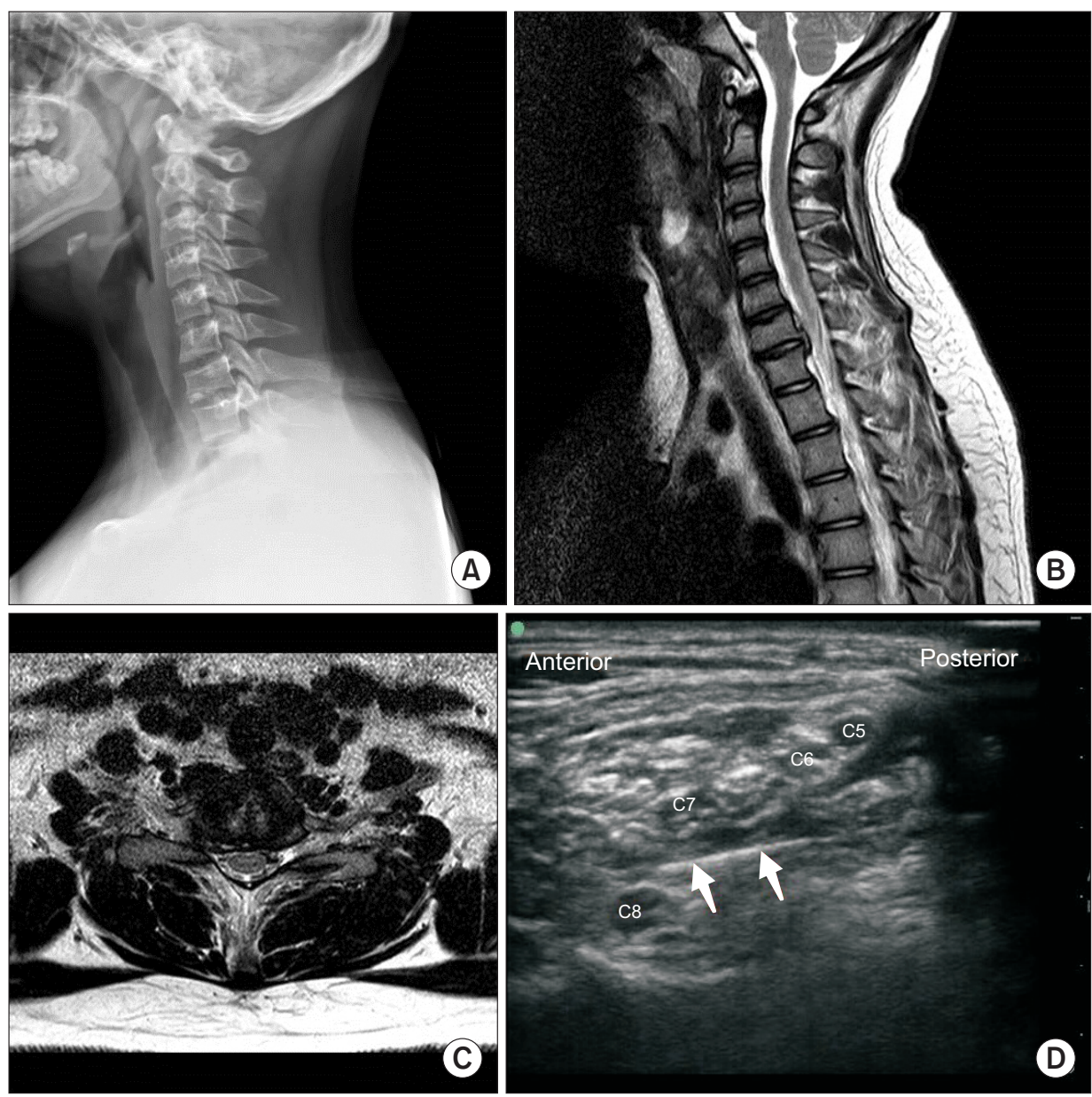

Fig. 1. Radiological image of the cervical spine and ultrasound image of $\mathrm{C} 8$ nerve root block. Lateral X-ray image (A) and sagittal (B), and axial (C) MRI show C7T1 intervertebral disc calcification (IDC). This IDC caused C8 nerve root compression. Four ventral rami (C5, C6, C7, and C8) and nerve stimulating needle are seen in the ultrasound image (D). Arrow, a blunt nerve stimulating needle. C5: 5th cervical nerve root, $\mathrm{C} 6$ : 6th cervical nerve root, C7: 7th cervical nerve root, C8: 8th cervical nerve root.

the calcified cervical disc.

The etiology is unclear. Laboratory tests show mostly normal results or moderate inflammatory syndrome [1]. There were non-specific findings in the study case. Radiographs reveal oval or round shaped calcifications that are most often located in the middle of the disk, but sometimes occupy the entire disk. MRI shows regions of reduced signal intensity on both, the T1- and the T2-weighted images, reflecting low proton density of the herniated calcific nucleus pulposus and its relation with myelin and the nerve roots. Rapid confirmation with MRI is very critical when the patient has neurologic symptoms.

The symptom disappears within one month, in most cases. Calcification regresses over the course of months to years, but may linger longer without symptoms. IDC is normally a benign condition. Majority of cases are treated conservatively and have good outcomes [2]. Therefore, the treatment of choice is conservative and symptomatic. It includes analgesics, nonsteroidal antiinflammatory medication, muscle relaxants, cervical soft collar, and limited physical activity [1]. The use of corticosteroids therapy has not been discussed in literature to date. IDC is known to be self limited with favorable results on conservative treatment, as described above. Severe pain, uncontrolled by medication, with successful interventional treatment such as CRB, has not been previously reported. Surgery is required in patients who develop progressive neurological deficit $[1,3]$. A decompression of the spinal cord with an anterior cervical diskectomy and an anterior fusion is usually required. Few cases in literature comment on recurrent neurological symptoms after surgical treatment [1].

Surgical treatment is a good approach for the short term, but it has not been scientifically validated by long term observation of symptoms $[3,4]$. It is based on the unique anatomic features of the pediatric spine. Curvature forms at age 10, and the adult skeleton completes from then on [5]. Therefore, careful surgical consideration of the effect on the biomechanism in the postadult spine is necessary. The results from a study with long-term observation of 381 young patients with surgery, showed degenerative changes at the adjacent level, and affected curvature [5].

Conservative management is currently the main treatment modality. However, in situations of uncontrolled pain in patients with IDC, CRB with ultrasound guidance is recommended for good results. 


\section{References}

1. Harvet G, De Pontual L, Neven B, Mary P, Letamendia Richard E, Nathanson M, et al. Paediatric intervertebral calcifications: two cases report and review of the literature. Arch Pediatr 2004; 11: 1457-61.

2. Imagama S, Wakao N, Ando K, Tauchi R, Tsuboi A, Ishiguro N. Conservative treatment for cervical radiculopathy due to extensive foraminal disc calcification in children. J Bone Joint Surg Am 2011; 93: e93 1-5.

3. Sato K, Nagata K, Park JS. Calcified intervertebral disc herniation in a child with myelopathy treated with laminoplasty. Spinal Cord 2005; 43: 680-3.

4. Li M, Rong W, Pan X, Yu L. Single-level calcified cervical disk herniation in a 13-year-old girl. Orthopedics 2012; 35: e1297-301.

5. Goffin J, Geusens E, Vantomme N, Quintens E, Waerzeggers Y, Depreitere B, et al. Long-term follow-up after interbody fusion of the cervical spine. J Spinal Disord Tech 2004; 17: 79-85. 